\title{
L'épiderme et l'épidémie
}

\section{Sur l'enjeu de visibilité dans la construction de deux catégories minoritaires}

Melaine Cervera

Renaud Hourcade

\begin{abstract}
Cet article questionne les enjeux de la visibilité et de l'invisibilité dans la construction des catégories socio-politiques. En mettant en miroir le combat des Noirs contre le racisme et celui des malades du $\mathrm{VIH} /$ sida contre l'épidémie, nous défendons l'idée que les catégories socio-politiques minoritaires se façonnent et se maintiennent dans une lutte, en apparence paradoxale, pour la visibilité. Nous appuyant sur la définition classique que Goffman a donnée du "stigmate ", cette comparaison cherche à mesurer les effets d'un marqueur social «évident» comme la couleur de la peau, ou dissimulable, comme la maladie, sur les registres de la mobilisation collective. Nous relativisons l'importance de cette distinction, en suggérant que les deux groupes livrent une seule et même bataille pour leur reconnaissance en tant que minorité sociale, et en avançant que le succès de cette lutte passe par leur visibilisation dans l'espace social. Les personnes atteintes du VIH doivent ainsi inventer leur visibilité, mais dans un monde social qui tend à stigmatiser leurs pratiques «déviantes", tandis que les Noirs se trouvent devoir réinvestir une catégorie raciale existante, mais pour en subvertir les frontières et le sens. Cette visibilisation du groupe et de sa souffrance peut comporter le risque d'une stigmatisation accrue, mais ce risque est accepté dans la mesure où la catégorie créée est une catégorie de lutte : elle sert à appeler une intervention publique qui prenne en charge à terme la destruction du stigmate, emportant avec elle la catégorie.
\end{abstract}

Mots-clefs : minorités, catégories, noirs, sida, VIH

This article compares two social movements with the view to understanding how the stakes of visibility and invisibility impact the building of social categories. The case studies are the struggle of Black people against racism, and that of HIV/sida victims against the epidemic. It emerges that visibility plays a key role in framing the borders of a social minority. Relying on Goffman's canonical definition of "stigma ", we try to assess in a comparative fashion the implications of a "bideable 》 social stigma (disease) and of an "obvious 》 one (blackness) on the mobilisation strategies. We argue that in fact both groups fight in a comparable fashion, looking to enhance their visibility in the social space in order to obtain official recognition as a minority. Whilst HIV/ sida victims have to invent their visibility, they don't do so freely, as norms and values weigh on their social reputation. Black people seem to have 
no choice but to match the existent racial category - but with the opportunity to redefine its "thickness » and signification. We eventually argue that a social group has to make its borders and suffering visible to become a "minority ", but this is not without the risk of reinforcing its stigmatisation. Hence, such a strategy can only be understood if one conceives the created category as limited in time and made only for the purpose of struggle. With the help of institutions called to fight the oppression they suffer, the category eventuality seeks its own destruction.

Keywords: minorities, categories, race, Aids, HIV

\section{Introduction}

La notion de «visibilité » est parmi les plus troubles des sciences sociales. La multiplication récente de ses usages, en particulier sous la forme de l'expression «minorité visible», peut facilement faire passer ce terme pour allant de soi : est « visible » ce qui se voit et dont la vue a une importance sociale, soit essentiellement un caractère physique stigmatisant. La catégorie de «minorité visible » fait ainsi avant tout référence à des critères raciaux ou ethniques, dans la mesure où elle intéresse le terrain de la lutte contre les discriminations les plus courantes ${ }^{1}$. Mais rien n'oblige à limiter les termes de la visibilité à ses marqueurs incontrôlables, comme le phénotype, l'âge ou le sexe. Dès lors qu'on les dote d'un symbole, ou qu'ils peuvent se donner à voir d'une quelconque manière, nombre d'autres caractères personnels peuvent entrer dans le champ du socialement visible : la classe sociale (un langage, une tenue), la religion (une barbe, un voile, une croix... les « signes religieux ostensibles» dont parle la loi française de 2004 visant le voile à l'école), et même, à la limite, une orientation sexuelle (un parler, une tenue). Ainsi entendue, la visibilité déborde vers le faire-savoir et le laisservoir, plus ou moins volontaires, et autorise à penser l'existence de visibilités ou de dissimulations stratégiques. Nous proposons dans cet article d'étudier l'importance de ce jeu de visibilité, subi ou volontaire, dans la construction des catégories sociopolitiques, et spécialement dans les catégories relevant des «minorités ». Associée au terme de minorité, la notion de visibilité appelle en effet des interrogations particulières, dans le sens où il n'est pas certain que la visibilité ne soit pas de toute façon nécessaire à définir une minorité, ou autrement dit, il n'est pas sûr qu'il existe des minorités invisibles. Depuis Fanon, on ne doute plus, par exemple, que le majoritaire est celui qui ne se voit pas, car si, conceptuellement, tout le monde a un sexe, une race ou une ethnie, sociologiquement, le dominant est le groupe qui a la capacité de nommer autrui sans avoir à se désigner, celui qui « conserve pour lui-même la généralité psychologique

1 L'expression est utilisée pour la première fois en 1995 au Canada dans la «Loi sur l'équité matière d'emploi ». 
et sociale » (GUILLAUMIN 1972, p.87). Si la majorité est toujours invisible, est-il juste de dire que, par effet de symétrie, la minorité sera toujours visible ? C'est au terme d'un rapport de force qui fixe à la fois les frontières du groupe, son signifiant et son signifié que se matérialise la perception du minoritaire. Historiquement, le terme s'applique à la différence ethnique dans un cadre impérial. Mais dès lors qu'il est le produit d'un processus généralisable, rien ne s'oppose à faire entrer dans l'analyse du «fait minoritaire » l'ensemble des rapports de pouvoirs politiques et sociaux mettant en jeu une définition de la différence collective : le sexe, la race, l'ethnicité, la classe sociale, la santé, l'âge, l'orientation sexuelle... La minorité n'existe qu'en tant que processus de « minoration » : la minorité « totale » est « celle qui est moins sur le plan numérique, qui est moindre sur le plan socioculturel, qui est mineure sur le plan sociétal et qui est minorisée sur le plan politique » (LEMIEUX 1986, p.10). Il faut tenter de comprendre ce qui fonde ce regard social sur la minorité, la nature des luttes sociales qui le façonnent autant que son contenu (une palette de degrés de perception du «hors-norme », allant du mépris à la reconnaissance). Ces deux aspects, qui doivent se penser ensemble, font l'objet des observations de cet article, qui cherche à comprendre comment un groupe peut se constituer en minorité, à quoi cela lui sert, et quel est le rôle de sa visibilité dans un tel processus. Deux luttes sociales contemporaines «minoritaires» servent de support à cette analyse : la mobilisation des Noirs et celle des personnes vivant avec le VIH $(\mathrm{PvVIH})$, dans une démarche qui voudrait emprunter la voie tracée par E. Goffman (1975, p.170) selon qui «l'élaboration de points de vue analytiquement cohérents sur les personnes stigmatisées ne saurait guère être le fait de ceux qui limitent leur intérêt à un seul des domaines [des études sociales]». Attentifs au "point de recoupement» de ces deux cas d'étude, nous suggérons que la catégorisation en tant que minorité sociale ne se réduit pas à une infériorisation venue d'en haut, mais qu'elle est aussi une mise en statut social, une forme de reconnaissance. De ce point de vue, on doit considérer que la catégorie de «minoritaire» se conquiert, qu'elle n'est pas uniquement et toujours subie mais peut, au contraire, faire l'objet d'une volonté et d'une action. Dans cette perspective, nous estimons que la lutte pour la visibilité joue nécessairement un rôle pivot : elle permet d'ériger un ensemble de personnes victimes d'une commune oppression en une minorité apte à lutter en tant que groupe, et à s'ouvrir, en tant que catégorie socio-politique défavorisée, au bénéfice de politiques publiques dédiées. Deux ordres de questions doivent être affrontés si l'on veut tirer tous les enseignements de ce processus. Par quoi passe la mise en visibilité d'une minorité ? Emprunte-t-elle les mêmes chemins et obéit-elle aux mêmes contraintes qu'il s'agisse, pour reprendre les catégories de Goffman (1963), d'un groupe discrédité (les Noirs) ou d'un groupe discréditable (les personnes atteintes du VIH) ? Pourquoi cette demande de reconnaissance en tant que minorité est-elle jugée plus importante que le risque de stigmatisation associé au supplément de visibilité qui est recherché ? 


\section{Régime de visibilité et stigmatisation}

Au-delà de leurs différences, une même injonction paradoxale semble peser sur les luttes sociales des Noirs et des porteurs du VIH, celle de se rendre visible en tant que groupe soumis à un traitement illégitime. Goffman (1975, p.64) définit la visibilité d'un stigmate comme «sa plus ou moins grande aptitude à produire le moyen de faire savoir qu'il est possédé par tel individu ». Si la visibilité du stigmate racial semble aller de soi, tout le sens de la lutte des Noirs est d'en réinterpréter les significations et la portée, dans une entreprise de constitution d'une catégorie non plus entièrement subie, mais en un sens «négociée » dans l'espace des relations sociales. En dépit de l'invisibilité sociale de leur affection, il semble que la problématique se présente dans des termes tout à fait comparables pour les $\mathrm{PvVIH}$. Si leur catégorie est presque entièrement à construire, elles n'en affrontent pas moins un terrain social semé de normes, de valeurs, et de déviances associées qui s'imposent à elles au moment de «choisir» leur visibilité. Un enjeu commun des deux luttes consiste donc à subvertir, en fonction des intérêts des minoritaires, les régimes du visible et de l'invisible.

«Accepter l'hospitalisation, c'est s'exposer à des regards incontrôlés sur soi, c'est se soumettre au langage, au rythme, aux intrusions des autres : on ne ferme plus sa porte à clé. Au pied du lit, des chiffres et des courbes, des listes de médicaments sont votre nouvelle carte d'identité. » (DE CECCATY 1994, p.86)

Le VIH, identifié au tout début des années 1980, s'est rapidement diffusé parmi les populations dites «à risques ». À la fin de l'année 1989, $79 \%$ des cas de sida déclarés concernaient la communauté homosexuelle masculine, concentrés dans la région parisienne (31 \% des diagnostics) (BROQUA 2005, p.8). La catégorie coutumière construite et utilisée au début des années 1980 est celle des « quatre H » (Homosexuels, Hérö̈nomanes, Hémophiles, Haïtien), témoignant de l'assimilation quasi-immédiate du sida («cancer gay»), dans l'imaginaire collectif, à des pratiques « déviantes». Un tel étiquetage vise, pour Becker (1985, p.32), «ceux qui transgressent les normes » et consiste à « rechercher dans la personnalité et dans les conditions de vie de ceux-ci les facteurs susceptibles de rendre compte de leur transgression ». Les PvVIH ont souffert et souffrent encore de ces représentations qu'a emprunté la construction initiale du " problème sida ». Même si la condition de la PvVIH demeure sans effet direct à partir du moment où l'affection reste non révélée, elle est génératrice de discrédit dans la mesure où l'image du VIH véhiculée socialement est teintée de valeur négative. Pour reprendre les termes goffmaniens, si l'individu conserve son identité réelle sans cheminer dans des processus d'appartenance et sans s'ancrer dans des pratiques désignées comme déviantes, il sera protégé par l'identité virtuelle construite dans l'interaction ordinaire avec son environnement. Mais dans les faits, le stigmate VIH est prégnant même pour le porteur qui le tait, car la séropositivité est imprégnée socialement du sentiment de culpabilité : on serait moins victime du VIH que du cancer, par exemple (cf. LANGLOIS 2006 ; LHUILIER, AMADO, BRUGEILLES \& ROLLAND 2007). Dans le cas du sida, l'accession du stigmate à la «notoriété » 
(GOFFMAN 1975, p.65) fait pénétrer son porteur dans un monde de perceptions infamantes, dans la mesure où la construction de la catégorie des PvVIH est façonnée par des représentations préexistantes relatives à l'homosexualité et à la toxicomanie. Une PvVIH révélant le secret de sa pathologie indique à son interlocuteur son appartenance probable à de telles catégories, qui structurent «l'image publique de la maladie, de ses victimes et des risques de contagion» (FILlieule 2001, p.213). Cette «notoriété », qui devient une évidence dans le milieu hospitalier, favorise en pratique le rejet et le mépris envers les malades :

«A l'hôpital je soignais des gens qui mourraient du sida (...). Y'avait quand même le comportement de mes collègues de travail, qui arrivaient dans les chambres masquées. Quand je travaillais, j'ai vu encore des gens qui jetaient les plateaux quoi. Qui ne les touchaient même pas, qui jetaient les plateaux repas comme ça quoi! Donc, il y avait un comportement très dur envers les malades. » (Entretien, PvVIH, 29 septembre 2009.)

Porteuses d'un stigmate invisible les rendant potentiellement «discréditables », les PvVIH semblent prisonnières d'une pression à la dissimulation. Cette injonction au maintien du secret implique qu'elles mettent en œuvre des stratégies de «contrôle de l'information » les concernant, évaluant à chaque interaction avec de nouveaux acteurs la possibilité ou non de dévoiler le secret du VIH. Or, «il n'existe aucun secret, si profond soit-il, qui ne soit connu de quelqu'un au moins, et qui donc ne puisse jeter une ombre» (GOFFMAN 1975, p.92). La problématique de la dicibilité du VIH, et à partir d'elle de sa visibilité incontrôlée, se place donc au cœur de la vie sociale des personnes infectées. Le risque prépondérant est du domaine de l'exclusion : stagnation professionnelle ou perte d'emploi, difficultés affectives et familiales ${ }^{2}$. Aussi ce risque ne peut-il être confronté que collectivement, et c'est à ce besoin de collectif que les associations de malades viennent répondre. En un sens, la mise en visibilité du stigmate dans la sphère publique ne peut raisonnablement s'opérer que sur le mode de la revendication et de l'action collective. Le problème s'envisage-t-il différemment pour les Noirs, porteurs d'un stigmate visible en toutes circonstances ? Leur lutte se situe sur le même terrain de la présentation de soi en tant que groupe. La discussion sur le stigmate, la réévaluation de son contenu et de son sens, se placent au cœur des rapports de force. «Aucune chance ne m'est permise. Je suis déterminé de l'extérieur » écrit Fanon dans Peau Noire, Masques Blancs (1952, p.93), faisant le constat sensible d'un ordre postcolonial intimement racialisé. Son régime de justification idéologique a beau

2 L'enquête VESPA «VIH : Enquête sur les personnes atteintes», ANRS, 2005, est éclairante sur la « dicibilité » du sida : $100 \%$ des employeurs interrogés déclarent ne pas donner suite à un entretien d'embauche durant lequel une personne mentionnerait sa séropositivité, $71 \%$ des personnes interrogées qui travaillent n'ont pas informé leur employeur de leur séropositivité, 50 $\%$ n'en ont pas informé leur médecin du travail et $73 \%$ n'en ont pas informé leurs collègues. 
chanceler - il n'y a plus grand monde pour adhérer aux postulats du racisme biologique, par exemple - la race continue de structurer les rapports sociaux, et pas seulement dans l'ancien monde colonisé. Puisant dans les racines profondes de la traite négrière et de l'esclavage, renforcé dans la conquête impériale, le stigmate d'être noir subit des modulations selon les lieux et les époques (fort mais maladif dans l'esclavage (DoRLIN 2006), paresseux et naiff sous la colonisation, malhonnête et criminel dans l'Amérique contemporaine (WACQUANT 2005)) mais finit par s'agréger dans un discrédit général qui demeure une réalité indéniable des rapports socio-politiques dans la plupart des sociétés contemporaines. La relation raciale est même souvent, comme en France, un ingrédient discret mais structurant de la production d'une idéologie nationale (DoRLIN 2006 ; SAADA 2007). Catégorie en un sens «évidente » mais dont la désignation suscite souvent le malaise, les Noirs constituent donc le parangon du marqueur social : d'un signe extérieur immanquable censé le résumer, le groupe majoritaire déduit pour le minoritaire un traitement social différencié. Mais à quoi tient réellement cette visibilité ? Abordé de l'extérieur, la visibilisation est l'œuvre même du racisme, à l'occasion institutionnalisé dans des dispositifs d'action publique, surtout lorsqu'un régime juridique est en jeu. Que l'on pense aux difficultés taxonomiques qu'on posées très tôt les pratiques de métissage aux colonies, ou à la classification radicalement généalogique de «la goutte de sang noir» instituée par les lois Jim Crow aux Etats-Unis, il est clair qu'un Noir n'est pas « naturellement » plus visible qu'un porteur du VIH, l'identification des frontières du groupe supposant un intense travail social. C'est le succès de ce travail qui rend à la couleur aujourd'hui son caractère d'évidence sociale. Une évidence qui emprisonne doublement ses victimes, comme l'a très bien décrit W.E.B. Du Bois en parlant de «double consciousness». Au-delà de la «blessure que l'esclavage a faite à la réputation de l'homme noir comme être humain », il y a l'impression obsédante de celui-ci « de toujours se regarder à travers les yeux d'un autre, de juger son âme avec les lunettes d'un monde qui n'a pour lui que de la compassion amusée et de la pitié » (DU BOIS, 1997 : 38, notre traduction). Pris dans l'étendue de ses effets, le stigmate de l'homme noir se rend visible à lui-même et entérine sa propre infériorisation, ou comme l'exprime Françoise Vergès commentant Fanon (2005, p.53) : « la 'race' est devenue consubstantielle à la subjectivité de l'homme 'noir'». Faut-il alors vivre avec sa visibilité ? Le Noir que dépeint Boris Vian (1946) est né blanc de peau. Libéré du stigmate, il peut à loisir passer pour un Blanc social. Mais, ne restant jamais qu'un Noir à ses propres yeux, il fait sienne l'oppression qui touche ses proches, suggérant ainsi qu'il n'y pas d'échappatoire individuelle à la catégorie sociale. Pourtant, en Afrique comme dans les communautés noires d'Europe, le commerce des solutions chimiques de blanchiment - hydroquinone, corticoïdes, et dérivés mercuriels - prospère (NDIAYE 2008 ; EYMERIAU 2009), paraissant faire des cosmétiques un dernier recours quand les solutions collectives semblent inaccessibles. 


\section{Luttes socio-politiques et visibilités alternatives}

A la différence des porteurs du VIH, les Noirs n'ont pas à s'inventer comme catégorie sociale, ils en subissent au contraire l'évidence. Dès lors, toute la lutte sociale qui s'est peu à peu organisé, sur les différents territoires de leur oppression, a visé à déjouer cette catégorie, tantôt dans ses frontières, parfois seulement dans son contenu. En somme, là où les porteurs du VIH se sont organisés pour occuper un espace vierge de catégories (mais non de normes morales), la lutte des Noirs a consisté à redéfinir subjectivement le sens de leur groupalité : ne vaut-elle que pour ce que les «Blancs»y mettent ? Dans quel sens renverser le stigmate ? Le mouvement de la négritude, qui prend son essor dans les années 1930, est peut-être la mieux connue de ces entreprises. Il s'agit de cesser de se dénier, de s'accepter comme Noir, mais avec une histoire, une culture, dont Césaire, avec Senghor et Damas, veut démontrer l'universalité. La « négritude » assume l'héritage racial de l'histoire, mais conteste la position qu'elle assigne aux Noirs. Ce faisant c'est davantage vers le contenu de la définition qu'elle dirige ses armes littéraires et politiques, contre la domination et l'aliénation. Quant à la démarcation, elle l'accepte : «puisque le mot 'nègre' définissait, sans qu'il fût besoin d'en dire plus, l'être Noir aux yeux des Blancs, les Noirs le volèrent aux Blancs pour en contester le sens » (PROTEAU 2001, p.17). Aux Etats-Unis, depuis le début du XXème siècle, d'autres voix ont entrepris de redéfinir l'être Noir, mais divergent sur les fondations de cette « renaissance ». Du Bois invoquait essentiellement une solidarité de race (" en tant que race nous devons lutter par l'organisation raciale, par la solidarité raciale, par l'unité raciale », DU BoIs, 1997, p.183) quand d'autres, Marcus Garvey en tête, prirent le chemin d'une quête de l'Afrique. Le Noir ne serait-il pas, simplement, un Africain ? Il serait alors riche de valeurs, d'une fierté, et d'empires. Riche aussi d'un espoir, celui de retourner à sa civilisation, sous la forme d'une Afrique essentialisée. Retrouver la «terre sans mal », le plus sûr moyen d'éradiquer l'infériorisation raciale, est un réel projet prôné par Garvey que des groupes de Noirs américains tenteront de mettre en œuvre. Cette approche afro-centriste, toujours présente (FAUVELLE-AYMAR 2002), s'est nourrie à partir des années 1950 des travaux de Cheikh Anta Diop désignant l'Égypte antique, l'«Égypte Noire », comme une preuve de la grandeur de l'histoire africaine, et pointant la richesse d'une culture (unifiée) dont les occidentaux refuseraient de reconnaitre l'influence jusque dans leurs propres civilisations. Un tel discours ne va cependant pas sans susciter de réserves, sinon des condamnations franches. Paul Gilroy (2000), qui ne voit dans l'essentialisme noir ou africain qu'un racisme inversé, a proposé, depuis L'Atlantique noir (1993), de lui substituer un " humanisme post-racial», articulé sur une défense des croisements culturels, des métissages, dont l'Atlantique serait une scène majeure depuis le commerce triangulaire. La posture est partagée, en France, par des auteurs antillais, héritiers en rupture de la «négritude», qui se réclament du «toutmonde » (GLISSANT 1997) ou de la «créolité » (CONFIANT, BERNABE \& CHAMOISEAU 1989). Ces derniers refusent de suivre Césaire dans son «aliénation », l'accusant de ne 
pas percevoir le sens et la vigueur du monde Créole en tant qu'une identité à célébrer, qui ne se résume pas à la "négritude» mais donne au contraire des armes pour la contestation des Noirs en tant que race. Un seul et même objectif prévaut derrière ce morcellement apparent : celui de se montrer différemment, pour renverser, en dépit de la contrainte mélanique, les catégories sociales du visible. Malgré une différence de temporalité manifeste, la mobilisation contre le sida ne va pas dans un autre sens. Pas plus que le mouvement noir elle ne construit sur du vide - la maladie et la sexualité sont enserrées dans un tissu de normes aussi historiquement construites que l'est la couleur de peau (voir par ex. FOUCAUlt 1976, Histoire de la sexualité). Elle ne doit donc, pas moins que le mouvement noir, se livrer à un renversement du stigmate. L'association Act Up-Paris, créée en 1989 par trois journalistes militants concernés par le VIH, constitue en France l'exemple phare d'une telle stratégie, jugée la plus propre à susciter une réponse des pouvoirs publics et du monde médical face à l'épidémie. Qu'est-ce qu'un séropositif ? Leur action s'appuie non sur le thème pur de la maladie, mais se propose de prendre pied sur le terrain de la déviance. Ils s'affichent comme « homosexuels séropositifs » sur la scène publique, et bâtissent en un sens sur les mêmes bases que le «Black is beautiful» de Marcus Garvey. Cette démarche engage ce qui a pu être appelé une "réhomosexualisation et une repolitisation» du sida. Une telle option les oppose alors explicitement à l'association AIDES, qui ne souhaitait pas mettre en avant le rôle de l'homosexualité dans la construction du problème, afin de ne pas risquer d'associer séropositivité et homosexualité et ainsi de courir le risque de doubler le stigmate attribué aux PvVIH (BROQUA 2006, p.38). C'est donc bien davantage la figure du malade, une catégorie d'entendement politique et social qui préservait une charge positive (associée à la victime innocente méritant compassion et assistance) qui avait été privilégiée par AIDES, l'association s'efforçant de créer un environnement protecteur propice à l'échange entre pairs. Mais que ce soit pour l'une ou pour l'autre des associations, l'espace qui rend possible la mobilisation est bien la construction duale de l'identité du malade du sida et du séropositif : vivant avec le VIH et en bonne santé (porteur sain), la personne affectée n'est pas forcément malade du sida. C'est donc dans cet intervalle morbide, allongé aujourd'hui par la disponibilité de traitements efficaces, que les militants trouvent leurs raisons d'agir. Le parti d'Act UpParis est celui d'actions associatives médiatiques, teintées par des rapports de force sociaux, à partir d'un discours qui les érige en porte-parole des malades face aux injustices que leurs font subir les institutions publiques. La mise au jour dans l'espace public de leur propre séropositivité et de leur propre homosexualité est le moteur de la mobilisation des militants, guidés par l'idée que si rien ne permet de distinguer un séropositif dans la rue, il n'existe pas. Il s'agit donc, pour reprendre le propos de la négritude, de «cesser de se dénier». La mise en visibilité des malades, autant que de leur mouvement, se place ainsi en enjeu clé de la construction du problème public, et à travers lui, d'une catégorie d'action publique : les malades du VIH/sida. Comment exister - et se battre - en tant que minorité opprimée, sans signe extérieur de 
«groupalité »? Malgré les apparences, le problème est commun aux Noirs et aux porteurs du VIH, les premiers devant subvertir le sens d'un marquage imposé, quand les seconds doivent tout inventer. D'où une violence symbolique certaine, parfois une brutalité, dans l'affichage, le marquage, voire la stigmatisation assumée. À travers les «ZAP», les «Dying », les « Outing » ${ }^{3}$, il faut appeler une attention que rien ne « fixe » naturellement. Les slogans témoignent du registre choisi ( Colère $=$ Action »; « Silence $=$ Mort $» ;$ Action $=$ Vie »), de même que les actions, toujours visuellement marquantes, ou que les couleurs de l'association, qui font écho au discours (rose et noir). En prenant le contre-pied de l'ancienne figure du malade, Act Up-Paris gagnera rapidement sa reconnaissance dans la lutte contre le sida. Ils amorceront en outre un virage important dans les modalités de la lutte contre le sida, en donnant aux PvVIH et aux malades euxmêmes le rôle de livrer la bataille. Comme l'analyse Broqua (2005, p.85) :

«La question du témoignage constitue un point de rupture entre anciennes et nouvelles associations ; les nouvelles associations de séropositifs apportent témoignages et expression de la séropositivité dans l'espace public alors que les précédentes (dont AIDES) préservent leurs 'usagers' de la curiosité des débats. »

Vingt ans plus tard, ce tournant des années 1980 - 1990 fait toujours débat au sein de la lutte contre le sida. La question de la visibilité est sans cesse reposée, par exemple lorsque les PvVIH témoignent à visage masqué lors des reportages diffusés par de grandes chaînes nationales au moment des rendez-vous de la lutte contre le sida (Sidaction TV, journée mondiale de lutte contre le sida). À l'instar du mouvement noir, les modalités et les enjeux de la visibilité ne sont pas figés mais font au contraire l'objet d'une transaction permanente. Ils doivent évoluer sous la pression d'organisations ou de discours concurrents, ou quand s'imposent de nouveaux référentiels (comme la diversité culturelle ou l'acceptation croissante de l'homosexualité). Le cas du sida laisse ainsi entrevoir deux régimes de visibilité concurrents, l'un qui s'oriente vers le « rassemblement d'individus qui doivent faire corps pour pouvoir énoncer une parole de nature collective » (BROQUA 2005, p.86), plus visible dans l'espace public, l'autre qui favorise les espaces de sociabilité protégés, étouffant la stigmatisation, mais qui ne donne peut-être pas au groupe les mêmes capacités d'interpellation des pouvoirs publics en tant que minorité agissante ${ }^{4}$.

3 «Zap » : interpellations spectaculaires d'institutions publiques, « Dying » : morts symboliques en pleine rue, «Outing » : dévoilement de l'homosexualité et/ou de la séropositivité d'un personnage public.

${ }_{4}^{4}$ Les années 1990 ont cependant pu laisser place à des stratégies communes: depuis 1994, le collectif TRT 5, acteur central de la lutte contre le sida sur la négociation des traitements et des essais thérapeutiques, rassemble sous la même bannière AIDES et Act Up-Paris, s'unissant pour faire valoir leur pouvoir de négociation avec l'institution. 


\section{Le détour par l'institution}

Comme l'écrit Pap Ndiaye (2005, p.91), un paradoxe veut que «les Noirs de France soient individuellement visibles, mais invisibles en tant que groupe social». Pour les Noirs comme pour les porteurs du VIH, c'est bien de cette mise en visibilité en tant que groupe, et précisément en tant que minorité, dont nous souhaitons rendre compte. À ce titre, une démarche apparaît commune aux deux mobilisations : la mise en catégories socio-politiques à travers un appel aux institutions. Si la visibilité sociale se révèle un enjeu structurant de la lutte pour la "groupalité », la demande d'une intervention publique, matérialisée dans des dispositifs spécifiques (soit la visibilité « institutionnelle»), apparaît ici comme une étape importante de la constitution des deux groupes en catégories minoritaires.

\subsection{Devenir une catégorie socio-politique}

Dans le rapport à la reconnaissance institutionnelle, la bataille première se livre pour le chiffre. Il est important de pouvoir se compter, mais davantage encore d'être comptés. Pour les PvVIH, et pour les Noirs exactement de la même manière, la mise en catégorie, dans sa dimension institutionnelle, occupe d'abord ce terrain du dénombrement. Il faut rendre visible la souffrance, délimiter ses contours statistiques, dresser la part numérique du fait minoritaire. Interpellé par les associations, et inquiet du danger sanitaire que présente l'émergence d'une épidémie inconnue, l'État cherche rapidement à récolter les chiffres, donnant dès lors à la maladie une visibilité statistique. Aujourd'hui, nombre d'agences sanitaires publiques (Institut National de Prévention et d'Éducation pour la Santé, Institut National de Veille Sanitaire) évaluent l'ampleur de l'épidémie selon différentes cibles, différentes pratiques et différentes catégories de la population. La lutte proprement dite se déploie à partir de 1989, avec la création du Conseil National du Sida, de l'Agence Nationale de Recherche sur le Sida et de l'Agence Française de Lutte contre le Sida. En moins d'une décennie, la lutte contre le sida se dote donc en France d'institutions propres, actionnant la visibilité institutionnelle de l'épidémie et de ses victimes. Militants et pouvoirs publics interagissent pour en orienter les termes : la prise en charge étatique s'agence sur des normes sociales que les associations s'efforcent de subvertir. En témoigne le travail d'Act Up autour de la catégorie de "séropositifs homosexuels». Mais la bataille pour les frontières de la catégorie, comme pour son contenu, n'a pas de fin, et cette figure particulière apparaît aujourd'hui diluée dans la catégorie plus générale de PvVIH ou de malade du sida. Cette lutte relève d'une concurrence entre groupes militants, comme le montre la divergence entre AIDES et Act Up, mais comme en témoigne également la démarche de certains Noirs de France cherchant à imposer une vision non-essentialisée de la race contre des conceptions plus substantialistes. Certes, depuis la Négritude, se rendre visibles dans l'espace public en tant que Noirs n'est pas une nouveauté. Mais les prémisses 
idéologiques de la démarche se démarquent radicalement de l'essentialisme qui prévalait jusqu'alors : ce qui réunit les Noirs est le racisme, une situation de commune oppression. Et c'est la lutte contre le racisme qui justifie que le groupe qui en est victime reprenne les frontières que les échanges sociaux lui donnent. Être Noir, dans cette dimension, est une identité raciale, parce que le racisme, et donc la race, sont une réalité sociale. Pap Ndiaye, théoricien et acteur du mouvement qui a donné naissance en France au Conseil représentatif des associations noires (CRAN) en 2005, se réfère à la distinction de Shelby entre «identité fine» et «identité épaisse»: la première tient simplement les Noirs pour des personnes qui n'ont rien d'autre en commun que « des traits physiques hérités tels que la peau noire, les cheveux crépus, un nez large et plat, et des lèvres épaisses. Quel que soit leur bagage culturel, c'est la catégorie de différence qui leur est imposée » (SHELBY 2002, p.239). L'identité «épaisse» y voit au contraire quelque chose de plus, un patrimoine biologique fixe, une origine culturelle ou des références communes qui fondent la solidarité des Noirs en tant que groupe. Le CRAN fait sienne la construction du groupe par la définition « fine » de l'identité imposée. Mais les frontières de la catégorie font parallèlement l'objet d'une négociation jamais terminée avec les institutions interpellées à l'extérieur du groupe. S'agissant du sida, le passage de la maladie mortelle à la pathologie chronique entraîne aujourd'hui, par exemple, la reconnaissance du VIH comme handicap, selon une définition légale qui y inclut, depuis 2005, le «trouble de santé invalidant» (cf. CELSE \& GEOFFroy 2009). Ce faisant, c'est toute la visibilisation du groupe, sociale et institutionnelle, qui évolue (JOURNAL DU SIDA 2009). Et avec elle se dessine un régime juridique renouvelé, débordant le champ propre du sida, en donnant accès notamment au statut de travailleur handicapé. $\mathrm{Si} \mathrm{à} \mathrm{un} \mathrm{niveau} \mathrm{individuel,} \mathrm{cette} \mathrm{reconnaissance} \mathrm{juridique} \mathrm{permet}$ l'insertion sociale de la $\mathrm{PvVIH}$, elle restreint néanmoins les possibles de l'action collective. L'immersion du VIH dans le prisme du handicap pose en effet la question de sa normalisation et de sa banalisation, taisant ainsi les spécificités revendiquées par les associations de lutte contre le sida et menaçant l'existence d'une catégorie sociopolitique distincte. Le mouvement Noir également, en se fondant sur la question du racisme et des discriminations, a jugé essentielle sa mise en catégorie d'action publique. L'urgence est-elle moindre que pour le sida ? Elle voudrait en tout cas passer par les mêmes voies : se rendre enfin visibles dans des statistiques officielles, qui, en France, ne veulent connaitre ni les « races », ni les religions, par l'effet d'une idéologie républicaine négatrice des identités particulières, doublée de préventions héritées de la seconde guerre mondiale. Mais surtout être traités en tant que catégorie d'action publique, s'ouvrant par sa situation particulière des droits spécifiques (discrimination positive, campagne de luttes contre les discriminations). C'est en se définissant autour d'un stigmate (réinterprété ou inventé) et d'une oppression (visibilisée) que les mouvements Noirs, exactement comme les malades du sida et les PvVIH, parviennent à se construire en tant que minorité souffrante. C'est le dialogue avec l'institution qui leur ouvre l'espace public en tant que catégorie socio-politique. 


\subsection{Se catégoriser pour se détruire ?}

Il faut constater que le rapport de force dans lequel s'inscrivent le CRAN et les autres associations de «visibilisation» de la cause Noire n'a, pour l'instant, pas permis de modifier le discours français. La race, même dés-essentialisée, n'y a pas gagné droit de citer. Si la lutte contre les discriminations s'est bel et bien dotée d'institutions propres (telle la Haute Autorité de Lutte contre les Discriminations et pour l'Egalité), les statistiques ethno-raciales demeurent en France des «données sensibles » au regard de la loi, et les rares autorisations accordées font souvent débat. Quant aux politiques de discrimination positive, elles restent confinées à des mesures expérimentales, ponctuelles, qui doivent contourner l'interdit de la race en se référant à des données socio-géographiques (à l'instar des mesures prises par Science Po Paris pour diversifier son recrutement). Le soupçon de la substance continue de peser sur les mouvements noirs, dont beaucoup craignent qu'en se présentant comme tels, ils ne contribuent finalement qu'à «faire vivre la race». Il faudrait donc ne pas rendre visible une oppression par crainte de l'entretenir. Les PvVIH, placés finalement devant un choix semblable, prennent aussi le risque du stigmate en s'engageant. Si leur mobilisation leur a fait gagner collectivement une reconnaissance institutionnelle et des moyens d'action qui font figure d'exemple dans l'avènement de la "démocratie sanitaire » (BATIFOUlier, DOMin \& GADREAU 2008, p.32), la tension avec l'intérêt individuel perdure. La PvVIH militante continue de courir le risque d'un discrédit quotidien, redoublé d'ailleurs par l'effet des politiques de prévention (PERETTI-WATEL \& MOATTI 2009, p.35), qui différencient la population en sous-catégories dites «à risques ». Les nouvelles problématiques que traverse aujourd'hui le champ de la lutte contre le sida, comme l'accès à l'emploi ou la question des publics migrants, peuvent en effet conforter une stigmatisation existante, dans la mesure où les politiques de réduction des risques de la transmission du VIH se retrouvent, d'une certain manière, à désigner des états déviants. La mise en catégories obéit donc à un effort de visibilisation sociale de la part de minorités souffrantes, tout en comportant le risque que des interprétations adverses se greffent sur le stigmate qu'ils ont construit. Qu'est-ce qui les emmène alors à prendre un tel risque ? Un enseignement important à tirer de l'analyse de ces deux mobilisations est le pouvoir prêté aux institutions, et à l'État, la première d'entre elles. Ces mouvements donnent foi à la capacité de l'État à faire disparaître, à terme, la catégorie qu'ils ont avec lui créée (ou qu'ils tentent de créer). L'objectif n'est pas de faire vivre pour elle-même une catégorie socio-politique, mais de forger des catégories de lutte politique, dans le but d'effacer progressivement, qui le stigmate lié au VIH, en faisant de ce virus une maladie comme les autres, qui le stigmate lié au phénotype, en faisant du noir une couleur de peau comme les autres. L'objet ultime de la lutte semble bien être l'invisibilité, sociale et institutionnelle. Cela peut certes sembler un horizon lointain pour le mouvement noir, mais il s'agit déjà d'une réalité pour les militants de la lutte contre le sida, les avancées contre la maladie, réelles depuis la mise sur le marché 
des trithérapies en 1996, ayant amorcé une démobilisation des PvVIH (BARBOT 2002, p.256). Pour le cas du sida, l'horizon qui s'ouvre, à mesure que les associations se saisissent de nouveaux terrains, est plutôt celui d'un éclatement de la catégorie, au profit d'anciens sous-groupes mis sur la voie d'une autonomisation. L'ontologie toujours relationnelle des minorités en fait des catégories labiles à l'infini, que les rapports de force sociaux, confrontant besoins visibilisés et actions publiques, construisent et reconstruisent en permanence.

\section{Conclusion}

« J'accepte ! La carte du monde faite à mon usage, non pas teinte aux arbitraires couleurs des savants, mais à la géométrie de mon sang répandu, j'accepte. Et la négritude, non plus un indice céphalique, mais mesurée au compas de la souffrance, j'accepte, j'accepte tout cela. »

Si Césaire (1983, p.56) se résigne, c'est à devoir lutter. C'est à l'obligation qui est faite au Noir, et à toutes les minorités, de porter le fer d'abord dans le champ de ce qui est rendu inexistant à force de n'être pas vu ou d'être mal mesuré. Qu'est-ce qu'être Noir? Qu'est-ce qu'être malade ? Où est la souffrance ? C'est en se désignant, et c'est en négociant, à la fois leurs limites, leurs acteurs et leurs sens, que des groupes sociaux font advenir les catégories d'appréhension du monde social. Leur réalité n'est pas autre chose que le fruit d'un rapport de force qui met aux prises des institutions et des mouvements sociaux (parfois contradictoires). Le visible et l'invisible, dans cette lutte, sont moins une caractéristique préalable que des outils, et ne sont en fait des minorités que les groupes qui se donnent à voir. C'est vrai pour les Noirs, dont la visibilité n'est paradoxalement pas une évidence. C'est vrai aussi pour les porteurs du VIH, qui choisissent de se dévoiler en sachant que le monde pensera qu'ils sont malades d'être déviants. Mais si la visibilité peut se désirer à l'intérieur d'un groupe, elle ne se justifie que si elle sert, à terme, l'éradication du stigmate. C'est dans cette perspective que le recours à l'État nous semble, au terme de cette réflexion, un enjeu structurant des luttes minoritaires. L'institution a les moyens d'agir, du moins les militants le croient-ils, qui sinon n'auraient que faire de s'ériger en catégorie d'action publique. Confrontée à une injustice, une lutte, un groupe, l'institution a la capacité de les rendre visibles, moins au sens visuel qu'au sens éthique de la reconnaissance. Axel Honneth (2006, p.271), articulant une réflexion majeure sur ce thème, écrit à ce propos que «lorsque de nouveaux modes de reconnaissance généralisés se sont imposés socialement, alors des dispositions juridiques doivent se transformer, d'autres modalités de représentation politique doivent s'établir et des redistributions matérielles doivent être entreprises ». Mais il faudrait réfléchir à retourner la proposition, et faire l'hypothèse que c'est parce que le sida a été doté d'institutions adaptées qu'il a accédé à une reconnaissance que l'invisibilité sociale lui déniait. À l'inverse, on pourrait alors soutenir que l'absence de 
réponse institutionnelle à la lutte pour un autre régime de visibilité que mènent certaines associations noires participe du maintien de rapports sociaux racialisés.

\section{Bibliographie}

BARBOT J. (2002): Les malades en mouvements. La médecine et la science à l'épreuve du sida. Paris: Balland.

Batifoulier P., Domin J.-P., Gadreau M. (2008): «Mutation du patient et construction d'un marché de la santé. L'expérience française ». In Revue française de socio-économie, vol. $1 \mathrm{n}^{\circ} 1, \mathrm{pp} .27-46$.

BECKer H. (1985): Outsiders, Etudes de sociologie de la déviance. Paris: Métaillé.

Broqua C. (2005): Agir pour ne pas mourir. Act Up, les homosexuels et le sida. Paris: Presses de la Fondation nationale des sciences politiques.

BUTON F. (2005): «Sida et politique: Saisir les formes de la lutte ». In Revue française de science politique, vol.55 n⿳0 5-6, pp.787-810.

CECCATTY (DE) R. (1994): L'accompagnement. Paris: Gallimard.

Celse M., GeOfFroy L. (2009): VIH, Emploi et Handicap : Avis suivi de recommandations sur la prise en compte des personnes vivant avec le VIH dans les politiques de handicap. Paris: Conseil National du Sida.

Cesaire A. (1983): Cahier d'un retour au pays natal. Paris et Dakar: Présence Africaine.

Confiant R., Bernabe J., Chamoiseau P. (1989): Eloge de la Créolité. Paris: Gallimard.

DORLIN E. (2006): La Matrice de la race. Généalogie sexuelle et coloniale de la Nation française. Paris: La Découverte.

Du BoIs W. (1997): The Souls of Black Folks. Boston: Bedford Books.

EPSTEIN S. (1996): Impure Science: AIDS, Activism, and the Politics of Knowledge. Berkeley: University of California Press.

EYMERIAU C. (2009): « S'éclaircir pour faire peau neuve ». In Corps, nº7.

FANON F. (1952): Peau Noire, Masques Blancs. Paris: Seuil.

FAUVELLE-AYMAR F.-X. (2002): «Naissance d'une nation noire. Multimédia, mondialisation et nouvelles solidarités ». In L'Homme, n¹61, pp.75-89.

Fillieule O., Broqua C. (2000): Les associations de lutte contre le Sida. Approche des logiques de l'engagement à Aides et Act Up. Paris: Rapport de recherche CEVIPOF/MIRE/Fondation de France. 
Fillieule O. (2001): «Proposition pour une analyse processuelle de l'engagement individuel. Post Scriptum ». In Revue française de science politique, vol.51 n¹-2, pp.199217.

Foucault M. (1976): Histoire de la sexualité. Paris: Gallimard.

GILroy P. (2000): Against Race: Imagining Political Culture Beyond the Color Line. Cambridge MA.: Harvard University Press.

GILroy P. (2003): L'Atlantique noir. Modernité et double conscience. Paris : Kargo.

GLissant E. (1997), Traité du Tout-Monde. Paris: Gallimard.

Goffman E. (1975): Stigmate. Les usages sociaux des handicaps. Paris: Editions de Minuit.

Guillaumin C. (1972): L'idéologie raciste. Genèse et langage actuel. Paris et La Haye: Mouton.

Honneth A. (2006): La société du mépris. Vers une nouvelle théorie critique. Paris: La Découverte.

JOURNAL DU SIDA (2009): "Passer le cap du handicap », n²13, octobre - décembre 2009.

Langlois E. (2006): L'épreuve du sida. Pour une sociologie du sujet fragile. Rennes: Presses universitaires de Rennes.

LEMIEUX V. (1986): «Les minorités et l'État: quelques propositions générales ». In Guillaume P., et al (1986): Minorités et État. Bordeaux et Québec. Presses universitaires de Bordeaux et Presses de l'université Laval, pp.9-20.

Lhuilier D., Amado S., Brugeilles F., Rolland D. (2007): «Vivre et travailler avec une maladie chronique (vih-vhc) ». In Nouvelle revue de psychosociologie, vol. $2 \mathrm{n}^{\circ} 4$, pp.123-141.

NDiAYE P. (2005), « Pour une histoire des populations noires. Préalables théoriques ». In Le mouvement social, $\mathrm{n}^{\circ} 213$, pp.91-108.

NDIAYE P. (2008): La Condition noire. Paris: Calmann-Lévy.

Peretti-Watel P., MoAtTi J-P. (2009): Le principe de prévention. Le culte de la santé et ses dérives. Paris: Seuil - La République des idées.

Proteau L. (2001): «Entre poétique et politique, Aimé Césaire et la 'négritude' ». In Sociétés contemporaines, $\mathrm{n}^{\circ} 44$, pp.15-39.

SAADA E. (2007): Les enfants de la colonie. Paris: La Découverte.

SEARLE J. (1995): La construction de la réalité sociale. Paris: Éditions de Minuit. 
SHELBY T. (2002): "The Foundations of Black Solidarity: Collective Identity or Common Oppression ». In Ethics, vol.112 n², pp.231-266.

Verges F. (2005): “'Le Nègre n'est pas. Pas plus que le Blanc.' Frantz Fanon, esclavage, race et racisme ». In Actuel Marx, n³8, pp.45-63.

Vian B. (Sullivan V.) (1946): J'irai cracher sur vos tombes. Paris: Éditions du Scorpion.

VOIROL O. (2009): «Les formes de l'invisibilité ». In Le temps philosophique, n¹3, pp.117140.

WACQUANT L. (2005): «La race comme crime civique ». In Revue internationale des sciences sociales, $\mathrm{n}^{\circ} 183$, pp.135-152. 